\title{
Performance Comparison of AODV and DSR on-Demand Routing Protocols for Mobile Ad-Hoc Networks
}

\author{
Prem Chand \\ Research Scholar \\ Department of CSE, MRIU \\ Faridabad, Haryana, India
}

\author{
M.K.Soni \\ E.D. \& Dean \\ FET, MRIU, \\ Faridabad, Haryana, India.
}

\begin{abstract}
A mobile ad-hoc network (MANET) is a collection of mobile wireless nodes, which communication to each other without any centralized control. These networks are characterized by a lack of infrastructure, and by a random and quickly changing network topology: thus the call for a strong dynamic routing protocol that can accommodate in such an environment is generated. In addition to this, routing protocols face many challenges like short battery backup, limited processing capability. This paper is an effort to study the performance of two on-demand routing protocols (AODV and DSR) in terms of number of routes selected, number of hop counts, number of RREQ packets and number of RREP packets. Simulation on QualNet shows that AODV compared with DSR reduces the number of hop count nodes, and AODV has less number of routes as compared to DSR, which helps AODV to be more efficient and less bulky. While comparing route request packets AODV is again better by having more number of RREQ packets as compared to DSR which made it more efficient in finding a new route and each time in replacing a stale link.
\end{abstract}

\section{General Terms}

This paper is effort to study performance comparison of two on-demand routing protocols for mobile ad-hoc networks with the help of QualNet simulator.

\section{Keywords}

Ad-hoc networks, Performance, AODV, DSR, Routing protocols.

\section{INTRODUCTION}

A Mobile Ad-hoc Network (MANET) [1] is an autonomous network that can be formed without any established infrastructure. As these networks are rapidly deployable and they don't rely on external infracture, it makes them an ideal candidate for rescue and emergency operations, military operations in the battlefield etc. Although all wireless networks as shown in Fig 1 work without any physical connection but with a fixed infracture. The MANETs also work without physical connection and also without any fixed infracture with the help of nodes having capabilities of sending and receiving data. This operation of sending and receiving data from one node to another is done with the help of routing protocols. The routing protocols [2] for MANET [3] can be categorized into two main types: reactive and proactive. In case of proactive protocols like DSDV [4], STAR and GSR the nodes in the ad-hoc network must keep track of all the routes to all other nodes. In case of reactive routing protocols such as DSR, AODV, ABR and SSA, a lazy approach is applied. The nodes do not keep the routes to all other nodes. Thus, there is no need of constant replacement [5] of routing information between nodes which results to save limited battery power of the nodes. To find out the routes to the destinations on demand flooding of route query packets on whole network is being done. In this paper we carry out a systematic performance [6] study of the two routing protocols for mobile ad-hoc network - Ad-hoc On Demand Distance

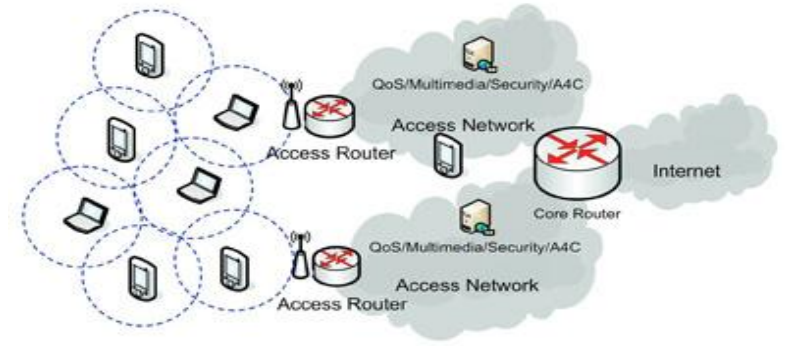

Fig 1. An example of various wireless networks

Vector Routing (AODV) and Dynamic Source Routing (DSR) protocol. We have used the means of simulation using QualNet 5.0(evaluation version) to gather data about these routing protocols in order to evaluate their performance.

This paper is ordered as follows. The Problem formulation and major issues are given in section 2 and simulation model and performance metrics in Section 3. We present the simulation results and analysis in section 4. Finally Section 5 concludes the paper and defines topics for further research.

\section{PROBLEM FORMULATION AND MAJOR ISSUES}

The key issue with ad-hoc networking [7] is how to send a message from one node to another with no direct link. The nodes in the network are moving around randomly, and it is very difficult that which nodes are directly linked together. Same time topology of the network is constantly changing and it is very difficult for routing process. A number or routing protocols are available at present; some of them are taken here for discussion purpose.

\subsection{Types of MANET Routing}

Nodes in MANET function as routers that discover and maintain routes to other nodes in the network. The primary goal in ad-hoc network is to establish a correct and efficient route between a pair of nodes and to ensure the correct and timely delivery of packets. The protocols for routing [8] can be classified as:

\subsubsection{Proactive/Table-Driven Routing}

Protocols: In proactive routing protocols, each node maintains routing information [9] to every other node in the network. The routing information is usually kept in a number 
of different tables. These tables are periodically updated and/or if the network topology changes. The difference between these protocols exists in the way the routing information is updated, and the type of information kept at each routing table. Keeping routes to all destinations up-todate, even if they are not used, is a disadvantage with regard to the usage of bandwidth and of network resources [10]. It is also possible that the control traffic delays data packets, because queues are filled with control packets and there are more packet collisions due to more network traffic. Proactive protocols do not scale in the frequency of topology change. Therefore the proactive strategy is appropriate for a low mobility network.

\subsubsection{Reactive/On-Demand Routing}

Protocols: These protocols were designed to overcome the wasted effort in maintaining unused routes. Routing information acquired only when there is a need for it. The needed routes are calculated on demand. This saves the overhead of maintaining unused routes at each node, but on the other hand the latency for sending data packets will considerably increase. It is obvious that a long delay can arise before data transmission because it has to wait until a route to the destination is acquired. As reactive routing protocols flood the network to discover the route, they are not optimal in terms of bandwidth utilization, but they scale well in the frequency of topology change. Thus this strategy is suitable for high mobility networks. Reactive protocols can be classified into two categories [11], Source routing and Hopby-hop routing. In Source routed on-demand protocols, each data packets carry the complete source to destination address. Therefore, each intermediate node forwards these packets according to the information kept in the header of each packet. This means that the intermediate nodes do not need to maintain up-to-date routing information for each active route in order to forward the packet towards the destination. Furthermore, nodes do not need to maintain neighbor connectivity through periodic beaconing messages neighbors through the use of beaconing messages. In hop-by-hop routing (also known as point-to-point routing), each data occurs by coding route request packets through packet only carries the destination address and the next hop address. Therefore, each intermediate [12] node in the path to the destination uses its routing table to forward each data packet towards the destination. Here we are discussing two on-demand routing protocols for MANET.

\section{A. The Ad-hoc On Demand Distance Vector (AODV)} routing algorithm is a routing protocol designed for ad-hoc mobile networks. It can perform both unicast and multicast routing. AODV [13] is an on demand algorithm, meaning that it builds routes between nodes only as desired by source nodes. Here the routes are maintained as long as they are needed by the sources. Furthermore, it forms trees which connect multicast group members. AODV uses sequence numbers [14] to ensure the freshness of routes. It is loop-free, self-starting, and scales to large numbers of mobile nodes. AODV makes routes by a route request / route reply message packets. When a source node desires a route to a destination for which it does not already have a route, it broadcasts a route request (RREQ) [15] packet across the network. Nodes receiving this packet update their information for the source node and set up backwards pointers to the source node in the route tables. Along with the source node's IP address, current sequence number, and broadcast ID, the RREQ also contains the most recent sequence number for the destination. A node may send a route reply (RREP) message after receiving the
RREQ if it is either the destination or if it has a route to the destination with corresponding sequence number greater than or equal to that contained in the RREQ. If this is the case, it unicasts a RREP back to the source. Otherwise, it rebroadcasts the route request message (RREQ). Nodes keep track of the RREQ's source IP [16] address and broadcast ID. If they receive a RREQ which they have already processed, they discard the RREQ and do not forward it.

B. Dynamic source routing (DSR) is an on demand routing [17] protocol which is designed for the purpose of multihop wireless networks. DSR contains two mechanisms of route discovery and route maintenance. The route discovery [18] phase initiate when source does not know route to the destination. Route cache [19] is also maintained for the purpose of storing old routes. When source sends a message to destination it first search it into the route cache if not found it generates a RREQ message and work in RREQ/RREP [20] fashion. The DSR protocol allows nodes to dynamically discover a source route across multiple network hops to any destination in the ad-hoc network. Each data packet sent then carries in its header the complete, ordered list of nodes through which the packet must pass, allowing packet routing to be trivially loop-free and avoiding the need for up-to-date routing information in the intermediate nodes through which the packet is forwarded. By including this source route in the header of each data packet, other nodes forwarding or overhearing any of these packets may also easily cache this routing information for future use.

2.1.3 Hybrid Routing The combinations of reactive and proactive protocols are called Hybrid protocols [21]. It takes advantages of these two protocols and as a result, routes are found very fast in the routing zone. Zone Routing Protocol (ZRP) is an example of Hybrid protocol.

\section{SIMULATION MODEL AND PERFORMANCE METRICS:}

We have used a detailed simulation model based on QualNet 5.0 (evaluation version), with GUI [22] tools for performance comparison [22]. The simulator contains standard API for composition of protocols across different layers. QualNet support a wider range of networks and their analysis, some of them are MANET, QoS [23], Wired Networks, Satellite and cellular.

We have primarily selected the following four performance metrics in order to study the performance comparison of AODV and DSR [24].

A. Number of route selected: This is defined as the number of routes offered by a routing protocol for an upcoming request.

B. Number of Hop count: This is defined as the number of intermediate nodes between a source and destination.

C. Number of route request packets (RREQ): This is defined as the number of route requesting packets used by a routing protocol to establish a connection between source and destination.

D. Number of route reply packets (RREP): This defined as the number of route replying packets as a result of RREQ packets.

\section{SIMULATION RESULTS AND ANALYSIS:}

For doing our analysis we have chosen some set of parameters to make the comparison between two exiting protocols. The Table 1 summarizes the simulation parameters that we have selected in order to evaluate the performance of the two routing protocols AODV and DSR; Simulation area size-1500 
x 1500; Mobility model-Random way point; Traffic typeConstant bit rate (CBR); Max speed-30m/sec.

Table 1. Simulator Parameters

\begin{tabular}{|l|l|}
\hline Configured Parameters : & 802.11 \\
\hline Physical Layer Protocol & AODV, DSR \\
\hline Routing protocol & Ro2.11.DCF \\
\hline Fading Model & Rayleigh \\
\hline Mobility Speed & \\
\hline Shadowing Model & Constant \\
\hline Energy Model & \\
\hline
\end{tabular}

\begin{tabular}{|l|l|}
\hline & \\
Application Layer & CBR Traffic \\
\hline
\end{tabular}

Our simulation experiments show the following different results for our four performance measuring parameters.

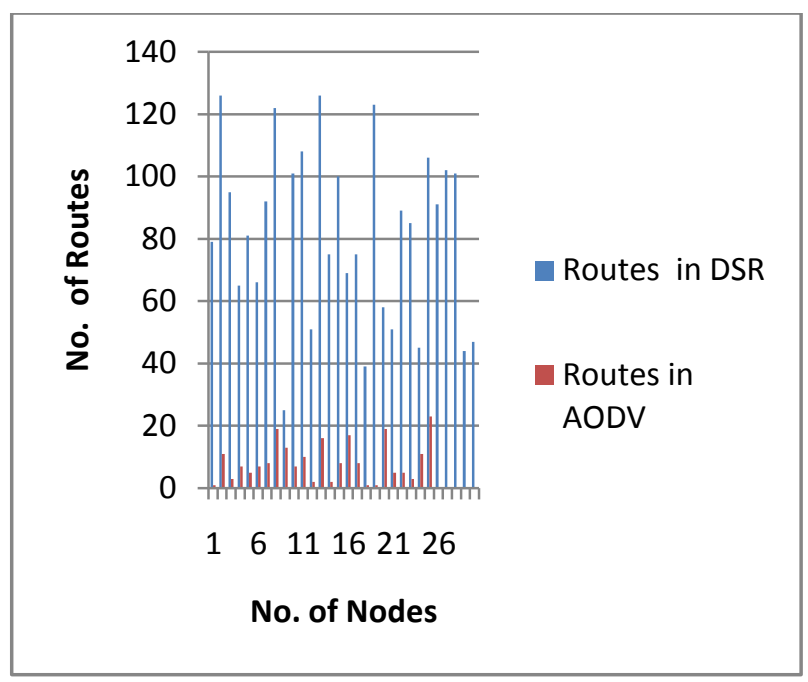

Fig 2. Number of routes selected by AODV and DSR

Fig 2. gives the comparison between Routes selected by both reactive routing protocols. Considering the various configured parameters it has been observed that the AODV routing protocol uses on demand approach for finding routes. The major difference between AODV and DSR stems out from the fact that DSR uses source routing in which a data packet carries the complete path to be traversed, while in AODV the source node and the intermediate node stores the next hop information corresponding to each flow data packet transmission.

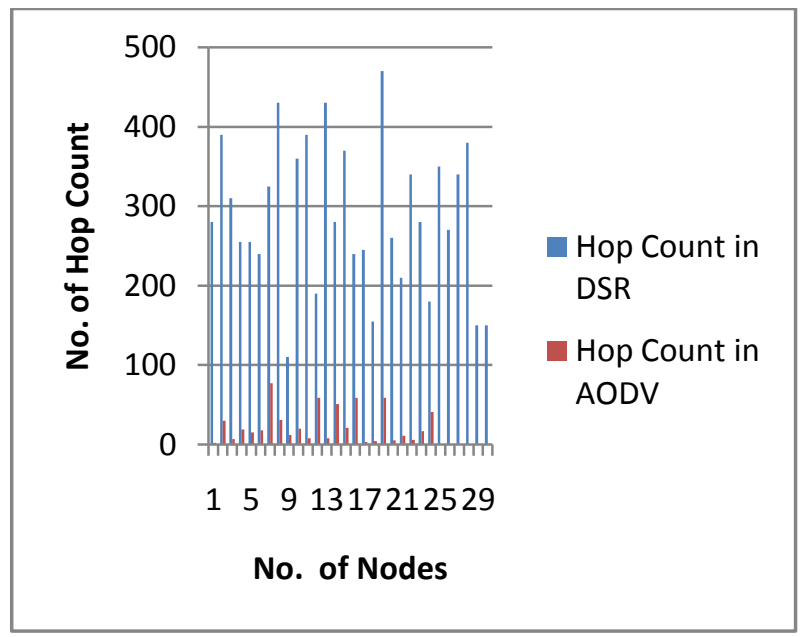

Fig 3. Comparison of Hop counts given by AODV and DSR

We see that AODV has less number of routes as compared to DSR, which helps AODV to be more efficient and less bulky. 
Fig 3 is the comparison of Hop counts chosen by AODV and DSR.

Here again we see that AODV has less number of intermediate (nodes between source and destination) nodes in comparison to DSR, which shows its efficient behavior as we know that more are the intermediate nodes more is the chance of path break and insecure network along with high energy consumption per message transfer by a node.

We have taken route request as the third comparison and are being shown in Fig 4.

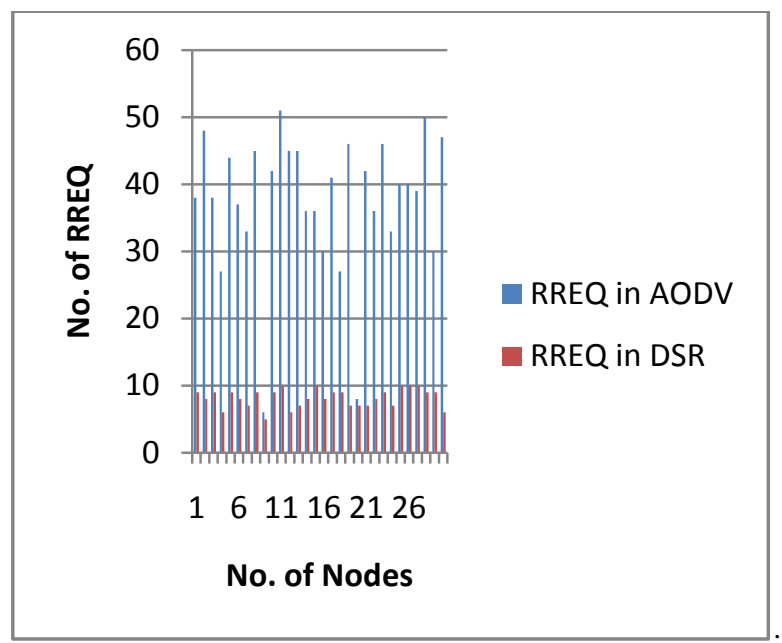

Fig 4. Comparison of route request packets in AODV and DSR

Comparing the route request made by AODV and DSR it is clear that DSR has less number of route request packets as compared to AODV, which made it less efficient in finding a new route and each time in replacing a stale link.

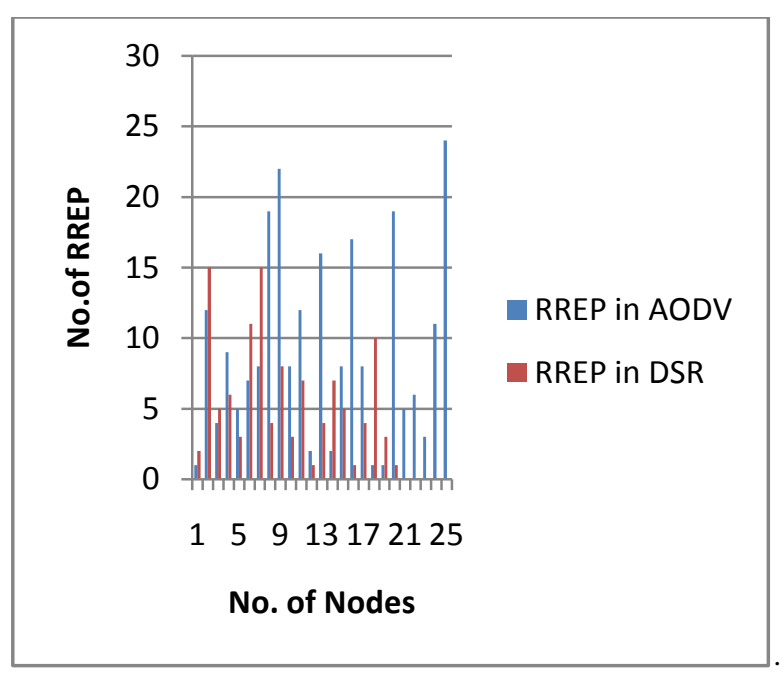

Fig 5. Route Reply packets in AODV and DSR

Fig 5 is the comparison of route reply packets made by AODV and DSR Here we see that AODV has more route reply options as compared to DSR; also DSR maintains multiple routes to the same destination in the cache. But unlike AODV, DSR has no mechanism to determine the freshness of the routes. It also does not have any mechanism to expire the stale routes. With high mobility, link breaks are frequent and there is the possibility of more routes becoming stale quickly. This requires the DSR to initiate the route discovery process which further adds to the increasing delay. From here also we can see that AODV is more efficient as compared to DSR.

\section{CONCLUSION}

The simulation results show that the performance characteristics of these two protocols with respect to route selection are better in case of AODV. Simulation results also indicate that DSR exhibits more intermediate nods in comparison to AODV. This is due to the fact that DSR being a source routing protocol, the initial path set up time is significantly higher as during the route discovery process every intermediate node needs to extract the information before forwarding the data packet. DSR has no mechanism to determine the freshness of the routes or to replace the stale routes. With high mobility link breaks will be frequent and thus there is the possibility of more routes becoming stale quickly. Simulation results also indicate that AODV has more RREQ and RREP options which made it more efficient as compared to DSR. In our future work, we plan to study the performance of these protocols under other network scenarios by varying the network size, the number of source nodes, the mobility models and the speed of the mobile nodes.

\section{REFERENCES}

[1] C. E. Perkins and E. M. Royer (1999): "Ad-hoc Ondemand Distance Vector Routing," Proc. 2nd IEEE Wksp. Mobile Comp. Sys. and Apps., pp. 90-100.

[2] M. Joa-Ng and I. T. Lu (1999): “A Peer-to-Peer ZoneBased Two-Level Link State Routing for Mobile Ad-hoc Networks". IEEE Journal on Selected Areas in Communications, vol. 17, no. 8, pp. 1415-1425.

[3] Hao Yang, Haiyun Luo. Fan Ye, Songwu Lu, and Lixia Zhang. "Security in mobile ad hoc networks: Challenges and solutions". IEEE Wireless Communications, February 2004

[4] Charles E. Perkins and Pravin Bhagwat(1994): "Highly dynamic Destination-Sequenced Distance-Vector routing (DSDV) for mobile computers". Proceedings of the SIGCOMM '94 Conference on Communications Architectures, Protocols and Applications, pages 234244 ,

[5] C. C. Chiang, H. K. Wu, W. Liu and M. Gerla(1997): "Routing in Clustered Multi-Hop Mobile Wireless Networks with Fading Channel". Proceedings of IEEE SICON 1997, pp. 197-211.

[6] P. Johansson, T. Larsson, N. Hedman, B. Mielczare, M. Degermark: "Scenario-based performance analysis of routing protocols for mobile ad-hoc networks", In Proc. of the ACM/IEEE MobiCom. (August 1999).

[7] T. H. Clausen, G. Hansen, L. Christensen, and G. Behrmann(2001): “The Optimized Link State Routing Protocol, Evaluation through Experiments and Simulation", Proceedings of IEEE Symposium on Wireless Personal Mobile Communications .

[8] A.Iwata, C. C. Chiang, G. Pei, M. Gerla, and T. W. Chen(1999): Scalable Routing Strategies for Ad-hoc Wireless Networks, IEEE Journal on Selected Areas in Communications, vol. 17, no. 8, pp. 1369-1379. 
[9] T. W. Chen and M. Gerla, (1998): "Global State Routing: A New Routing Scheme for Ad- hoc Wireless Networks", Proceedings of IEEE ICC, pp. 171-175.

[10] Charles Perkins and Elizabeth Royer(1999): "Ad-hoc ondemand distance vector routing". In Proceedings of the 2nd IEEE Workshop on Mobile Computing Systems and Applications, pages 90-100.

[11] C. K. Toh,(1997): "Associativity-Based Routing for Adhoc Mobile Networks". Wireless Personal Communications, vol. 4, no. 2, pp. 1-36.

[12] L. Chen and W.B. Heinzelman, "QoS-Aware Routing Based on Bandwidth Estimation for Mobile Ad Hoc Networks," IEEE J. Selected Areas in Comm., vol. 23, no. 3, pp. 561-572, Mar. 2005.

[13] Smt Rajashree.V. Biradar \& Prof V. C.Patil : Classification and Comparison of routing Techniques in Wireless Ad-hoc Networks, in the proceedings of international Symposium on Adhoc Ubiquitous Computing (ISHUC'06), pp. 7-11 (2006).

[14] C. E. Perkins, S.R. Das, and E. Royer, "Ad-hoc on Demand DistanceVector (AODV)". March 2000, http://www.ietf.org/internal-drafts/draft-ietf-manet-aodv05.txt

[15] R. S. Sisodia, B. S. Manoj, and C. Siva Ram Murthy(2002): "A Preferred Link-Based Routing Protocol for Ad-hoc Wireless Networks". Journal of Communications and Networks, vol. 4, no. 1, pp. 1421.

[16] P. Sinha, R. Sivakumar, and V. Bharghavan(1999), "CEDAR: A Core Extraction Distributed Ad-hoc Routing Algorithm". IEEE Journal on Selected Areas in Communications, vol. 17, no. 8, pp. 1454-1466.
[17] Z.. J. Haas (1997): “The Routing Algorithm for the Reconfigurable Wireless networks", Proceedings of ICUPC 1997, vol. 2, pp. 562-566.

[18] Rohit Dube, Cynthia D. Rais, Kuang-Yeh Wang, and Satish K. Tripathi(1997): "Signal Stability-Based Adaptive Routing (SSA) for Ad-hoc Mobile Networks." IEEE Personal Communications, 4(1):36-45.

[19] Y. C. Hu and D. Johnson (2000), "Caching Strategies in On-demand Routing Protocols for Wireless Ad-hoc Networks," Proc. IEEE/ACM MOBICOM '00, pp. 23142.

[20] David A. Maltz, Josh Broch, Jorjeta Jetcheva, and David B. Johnson(1999): "The Effects of On-Demand Behavior in Routing Protocols for Multi-Hop Wireless Ad-hoc Networks". IEEE Journal on Selected Areas of Communications, 17(8):1439-1453.

[21] Robert Castaneda and Samir R. Das(1999): "Query Localization Techniques for On-demand Routing Protocols in Ad-hoc Networks". In Proceedings of the Fifth International Conference on Mobile Computing and Networking (MobiCom'99). ACM.

[22] S.R.Das(1998): "Comparative Performance Evaluation of Routing Protocols for Mobile Ad- hoc Networks," 7th Int'l Conf. Comp. Commun. and Networks, pp. 153-61.

[23] Hanzo,II, L., Tafazolli, R., 2006, "Quality of Service Routing and Admission Control for Mobile Ad-hoc networks with a contention-based MAC Layer", IEEE International Conference on Mobile Adhoc and Sensor Systems, 9-12 October, 501-504.

[24] Per Johansson, Tony Larsson, Nicklas Hedman, Bartosz Mielczarek, and Mikael Degermark. Routing Protocols for Mobile Ad-hoc Networks-A Comparative Performance Analysis. In Proceedings of the Fifth International Conference on Mobile Computing and Networking (MobiCom'99). ACM, August 1999. 